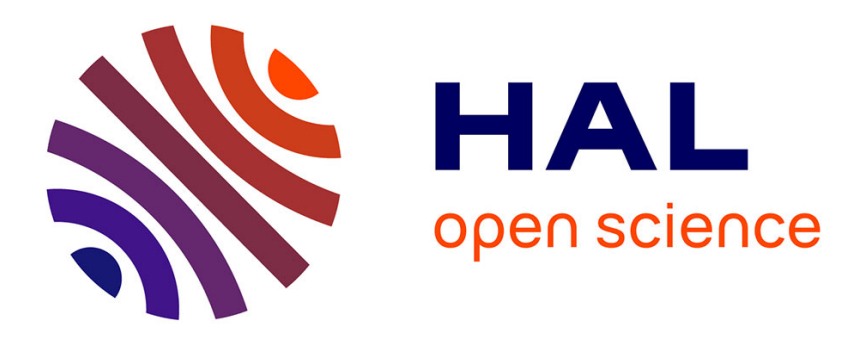

\title{
Le figural entre imagination et perception
}

Valeria de Luca

\section{To cite this version:}

Valeria de Luca. Le figural entre imagination et perception. Metodo. International Studies in Phenomenology and Philosophy, 2015, pp.199-220. hal-01163594

\section{HAL Id: hal-01163594 \\ https://hal-unilim.archives-ouvertes.fr/hal-01163594}

Submitted on 15 Jun 2015

HAL is a multi-disciplinary open access archive for the deposit and dissemination of scientific research documents, whether they are published or not. The documents may come from teaching and research institutions in France or abroad, or from public or private research centers.
L'archive ouverte pluridisciplinaire HAL, est destinée au dépôt et à la diffusion de documents scientifiques de niveau recherche, publiés ou non, émanant des établissements d'enseignement et de recherche français ou étrangers, des laboratoires publics ou privés. 


\title{
Le figural entre imagination et perception
}

\author{
Valeria De Luca \\ Université de Limoges CeReS (Centre de Recherches Sémiotiques) \\ v.deluca.83@gmail.com
}

\begin{abstract}
Whereas in semiotics we have tried to account for the activity of the figurative dimension of meaning as a coherent text deformation, in other knowledge fields, the study of figures of speech and of images, have led to an original design of "figural" as a full dimension of cultures. Whether it was a matter of the relationship between saying and seeing or of the nature and power of images, the "figural" has been designed as a device able to move the boundaries between: speech and desire, rhetoric and esthesia, reality and reference, between the subjectal constitution and the phenomenal donation, between the singular expression as semiotic praxis and stabilization of values over time.
\end{abstract}

\section{Sémiotique et méthode phénoménologique: l'épreuve figurale}

Si l'on se tourne vers l'histoire de la sémiotique de ces dernières vingt années, entendue autant comme discipline qu'en tant que méthode ou, en les termes d'Umberto Eco (1985), soit comme un ensemble des sémiotiques spécifiques, soit comme sémiotique générale, on a assisté, d'une part, à un véritable élargissement des champs ou domaines d'analyse, allant des passions et du corps aux pratiques sociales ayant engendré une pléthore de sémiotiques : de la photographie, du corps, des pratiques, etc. D'autre part, cette prolifération s'est partiellement rebattue sur une sémiotique plus générale, une voie d'investigation du sens du sens, un procédé d'interrogation de son faire ; par conséquent, au fur et à mesure que des « nouveaux " phénomènes réclamaient une description adéquate, sinon une explication, les modèles théoriques d'antan ont été soumis à une révision profonde, en constituant la sémiotique même comme une pratique (cf. Fontanille 2008). Le modèle génératif de la sémiotique greimasienne a notamment été modifié par l'intégration et la complexification des dimensions figurative et sensorielle-percpetive ;

Metodo. International Studies in Phenomenology and Philosophy

Vol. 3, n. 1 (2015)

ISSN 2281-9177 
néanmoins, un principe méthodologique ou, autrement dit, un impératif analytique, paraît demeurer, à savoir la possibilité de parcourir générativement des formes signifiantes, des phénomènes de signification une fois passés au crible de l'analyse et par là donc conçus comme des objets. Dès lors, un tel élargissement des champs d'analyse ainsi qu'une révision profonde d'une épistémologie structuraliste forte ouvrent de facto une brèche à la voie phénoménologique dans la mesure où la matière du sensible - entendu comme globalité des modes de donation/appréhension du sens en devenir - interroge les notions d'immanence et de transcendence non seulement par rapport aux expériences et aux vécus, mais également par rapport aux "êtres" faisant l'objet soit d'une analyse soit d'une description phénoménologique. En effet, comme le dit Eco, " une sémiotique générale est une philosophie des langages en ce sens qu'elle veut l'être non seulement par rapport aux règles de l'èrgon, mais également des processus de l'energheia. » ${ }^{1}$.

Dans les mailles intriquées de la sémiose, des ses matériaux - qu'ils soient de facture perceptive, cognitive, culturelle, etc. et pourvu que l'on puisse les dissocier - il y a toujours comme une sorte d'excès, de démesure - comme le dirait George Didi-Huberman -, de reste ou de lacune, de disponibilité, d'indétermination irréductible à une générativité descendant d'une délimitation d'un plan et d'éléments de base. En ces termes, le figural résiste d'un côté à nombre de modèles d'analyse et, de l'autre, à une conception trop stricte de l'immanence en sémiotique, en ré-affirmant précisément la nécessité de questionner cette energheia de la sémiose, cette énergie, cette force vivante par-delà son propre méta-langage qui, s'il est vrai qu'il construit et façonne son objet, ne peut guère se penser comme appartenant à un autre ordre de « réalité » - scientifique - et qui, par contre, ne peut se configurer que comme réflexion, processus, observation seconde sur le sens.

Le figural réside traverse les matériaux du sens, du discours à l'image, de la perception aux formes de vie, du geste aux émotions, en s'y incrustant, en s'y cachant, en s'y exprimant, en travaillant un « objet » quelconque jusqu'au cœur de son faire sens, c'est-à-dire en tant qu'activité qui disloque - et par le même coup reproduit et renouvelle - la différence et l'agonisme du jeu des valeurs. Il se constitue comme une durée, une survivance qui, afin d'être saisie, demande à ce que l'on se plonge, en la complexifiant, dans la dimension sensible de la signification, là où imaginaire et perception rendent visible l'émergence des formes signifiantes, là où l'on assiste non plus à une génération mais à une genèse de ces formes.

Par conséquent, il s'agira de traverser des zones d'indiscernibilité où le sens,

1 ECO 1985, 332.

Metodo. International Studies in Phenomenology and Philosophy

Vol. 3, n. 1 (2015) 
dans sa praxis, découvre les formes de sa propre teneur constituant les cultures ainsi que sa propre histoire.

\section{Figural et figuration : la sémantisation des figures chez Greimas}

Avant de convoquer certains auteurs ayant investigué la portée du figural dans le cadre d'une sémiotique plus "continuiste», phénoménologiquement orientée, où il n'y a pas de discrétisation entre perception et langage, entre sémiotique du monde naturel et sémiotique des langues naturelles - pour utiliser la formule du lituanien -, mais un enchaînement de formes de médiation - ce sur quoi nous reviendrons -, il convient d'éclaircir certains acquis et présupposés de l'élaboration greimasienne autour des concepts de figure et de figurativisation qui, par leur même façonnage, ont de facto écarté la dimension figurale des préoccupations de la sémiotique.

Loin de restituer pleinement la richesse et la profondeur de l'infrastructure théorique de Greimas par exigences de synthèse, nous tenons tout de même à remarquer des latences présentes dans la formulation de ces deux notions.

En ce qui concerne notre argumentation, nous nous attachons premièrement à l'examen des entrées «Figure» et «Figurativisation», telles qu'elles apparaissent dans le Dictionnaire rédigé avec Courtès, où, en sémantique discursive, les procédures de figurativisation consistent en l'investissement d'un objet syntaxique "permettant à l'énonciataire de le reconnaître comme une figure $»^{2}$, donnant ainsi l'impression référentielle par rapport aux figures du monde naturel. Ce passage est nécessaire dans la mesure où les figures du monde naturel, conçu lui-même comme une organisation sémiotique, "sont constituées par les 'qualités sensibles ' du monde » agissant "directement sans médiation linguistique sur l'homme ${ }^{3}$; de surcroitt, on lit dans le texte que les dites figures sont également repérables dans le plan du contenu des langues naturelles, pourvu que des sèmes extéroceptifs soient convoqués par le discours. En d'autres termes, cela revient à affirmer d'une part, l'autonomisation et l'abstraction des opérations s'effectuant tout le long du parcours génératif procédant des structures profondes aux structures de surface et, d'autre part, à sceller le socle, la discontinuité entre langue et perception.

Autrement dit, c'est parce que le sens, le sens reconstruit déductivement par

2 GREIMAS, COURTĖS 1979 : entrée « figurativisation ».

3 GREIMAS, COURTÈs 1979 : entrée « monde naturel ».

Metodo. International Studies in Phenomenology and Philosophy

Vol. 3, n. 1 (2015) 
le modèle d'analyse, se structure comme un parcours suivant une trajectoire allant des oppositions sémiques et des opérations syntaxiques de base jusqu'à ses multiples manifestations de surface et parce que, implicitement, une équivalence entre plan de l'expression et monde sensible s'installe, qu'il est besoin de concevoir les figures et la figurativisation comme une reconstruction, une représentation de ce même monde. Dans la mesure où ces qualités agissent directement et sans médiation linguistique, et dans la mesure où l'on postule des structures abstraites régissant la formation du sens, l'introduction de procédures de recouvrement figuratif s'avère nécessaire.

Cependant, ces procédures d'investissement, de conversion figurative ne sont pas anodines, se nichant dans le discours greimasien une sorte de préfiguration de la problématique figurale. En effet, toujours dans l'entrée «figurativisation» du Dictionnaire, on mentionne la figuration en tant que «mise en œuvre des figures sémiotiques ${ }^{4}$, dont le pendant est l'iconisation en tant que revêtement complet des figures. La figuration, telle qu'elle est décrite dans ce court passage, serait de prime abord une affaire de formes plutôt que des figures et par là même une telle opération concernerait non seulement les phases de réalisation des textes mais élargirait la réflexion à plusieurs formes sémiotiques et, par conséquent, à différentes substances. Revenant comme thème en filigrane dans De l'imperfection où l'auteur se consacr aux rapports entre perception et sémiose, la figuration concernerait, en dernière instance, comme l'affirment Fontanille et Tore, « la problématisation et la définition des sujets énonçants et des objets-énoncés ${ }^{5}$.

Toutefois, en dépit du caractère révolutionnaire de cette intuition, lorsque le lituanien aborde la figurativisation dans Sémiotique figurative et sémiotique plastique en envisageant la constitution d'un modèle d'analyse des textes visuels ou, à tout le moins, non verbaux, l'examen de la figuration comme mise en œuvre des formes sémiotiques ne trouve pas de place. En effet, dans ce texte visant à élaborer une grille de lecture du visuel, les figures se constituent en tant que telles à partir justement d'agencements de traits sémantiques intéroceptifs, et

c'est cette grille de lecture qui nous rend le monde signifiant, permettant d'identifier les figures comme des objets, de les classifier, de les relier entre elles $\lceil\ldots \mid$ De nature sémantique, - et non pas, par exemple, visuelle, auditive ou olfactive - elle sert comme « code» de reconnaissance rendant le monde intelligible et utilisable. On comprend [...] que c'est la projection de cette grille de lecture - une sorte de

4 GREIMAS, COURTĖs 1979 : entrée « figurativisation ».

5 FonTANILLE \& TORE 2006, 23-32.

Metodo. International Studies in Phenomenology and Philosophy

Vol. 3, n. 1 (2015) 
«signifié » du monde - sur une toile peinte qui permet de reconnaître le spectacle que l'on estime qu'il soit représenté.

La figuration précédemment évoquée cède sa place en faveur d'une lecture exquisément iconisante qui, en sélectionnant des traits sur le signifiant planaire et en leur attribuant des significations, transforme les figures en des « signesobjets », à savoir en des formants figuratifs. Le propre d'une telle lecture - dont l'abstraction n'est que l'autre pôle de l'iconisation - ainsi que du formant constitué, réside d'un côté dans le tri des faisceaux perçus et, de l'autre, dans la réduction de l'hétérogénéité, par exemple du visuel, à son homogénéisation eu égard de l'unité de la signification, de son cadrage, si l'on peut dire, dans la bonne forme. Par ailleurs, la comparaison avec la morphogenèse telle qu'elle avait été élaborée par la Gestalttheorie est repérable dans le texte, quelques lignes après l'étude des formants, lorsque l'on établi une convergence des deux approches autour des formes en tant qu' « unités discrètes ainsi constituées à partir de la somme de ses traits ${ }^{7}$. Ce parallèle, qui pourrait ré-ouvrir la réflexion et l'implémentation de la figuralité de et dans les formes sémiotiques s'arrête pourtant là, en demeurant sur des analogies de surface et en laissant inachevée cette piste. Celle-ci est également la raison pour laquelle, avant de revenir vers des nouvelles suggestions sémiotiques stricto sensu, il faut faire appel à ceux qui, en philosophie et en linguistique, ont approché le figural en toute sa richesse et complexité.

\section{Incommensurabilité du figural : de Lyotard aux formes sémantiques}

Tandis qu'en sémiotique l'on a cherché à rendre compte du travail de la dimension figurative du sens en tant que déformation cohérente des textes, dans d'autres champs du savoir l'étude des figures du discours ainsi que du visuel, a abouti à une véritable conception du figural comme dimension des cultures à part entière. Qu'il se soit agi des relations entre le dire et le voir ou bien de la nature et du pouvoir des images, le figural a été conçu comme un dispositif capable de mouvoir - d'émouvoir - les frontières entre la parole et le désir, entre la rhétorique et l'esthésie, entre la réalité et la référence, entre la constitution subjectale et la donation mondaine, entre l'expression singulière comme praxis sémiotique et la thésaurisation des valeurs dans le temps.

6 GREIMAS 1984, 34.

7 Greimas 1984, 38.

Metodo. International Studies in Phenomenology and Philosophy

Vol. 3, n. 1 (2015) 
Une véritable pensée du figural résulte débitrice du vaste héritage laissé par la notion de figure, figura - si l'on se tient à l'étymologie de la rhétorique ancienne -, dont il serait impossible de restituer ici toute son histoire . $^{8}$ Soulignons, pour ce qui concerne notre procédé, que, comme le dit bien Herman Parret, la figura est associée en latin à des significations telles "configuration, chose façonnée, manière d'être" et que, selon Auerbach, "le sens premier du mot est [...] celui de "forme plastique'" ; l'animation de la figura, notamment si l'on la rapproche de la forma, devient saillante en étant, toujours en suivant Auerbach, "en quelque sorte un opérateur intermédiaire qui fait passer de l'un [le moule de la forme] à l'autre par empreinte ${ }^{\prime 10}$, se situant dès lors dans un entre-deux, dans un interstice entre la conception imageante et l'incarnation corporelle.

C'est dans la lignée de ces élaborations que la théorisation du figural se dégage dans l'examen que Jean-François Lyotard en fait dans le célèbre Discours, figure en 1971. Bien qu'elle soit loin d'une pertinence sémiotique stricte, l'étude lyotardienne ouvre un champ d'investigation et pose des questions interpellant le faire même du sens. Son analyse vise à repenser les relations entre le dire et le voir, à savoir entre le discours et le visible et par là même l'art, à élucider les façons à travers lesquelles le figural travaille en tous les sens autant la langue que la peinture et finalement à considérer sous des nouveaux angles la dialectique entre transcendance et immanence innervant les chiasmes entre perception et langage, en mêlant les théories hégélienne et en particulier fregienne sur la signification jusqu'à une critique d'une certaine conception de la signification en sémiotique (notamment le modèle jakobsonien). Plus précisément, Lyotard reprend de Frege, en la problématisant et en maintenant en filigrane les questions de la vérité et de la constitution des sujets, la distinction entre Sinn - la signification d'un mot au sens de place occupée dans un système, à savoir la langue - et Bedeutung - l'acte de désignation d'une référence.

Dans les discours réalisés, dans tous phénomènes de parole, un écart, une épaisseur, se produisent entre ce que l'on juge appartenant à un ordre paradigmatique et ce qui relève du syntagmatique. Cet écart qui est en même temps, affirme Lyotard, la distanciation, l'acte de surreflexion - en reprenant une formule merleau-pontienne - entre le langage et le sensible, et du langage sur lui-même, fait entrevoir et trembler le mécanisme même de la langue car il trouble la constitution de l'invariance en mettant en mouvement une reprise

8 Pour une vue panoramique sur ses différentes acceptions, voir PARRET 2006.

9 PARRET 2006, 73.

10 Cité dans Aubral \& Chateau1999, 12 et 13.

Metodo. International Studies in Phenomenology and Philosophy

Vol. 3, n. 1 (2015) 
constante de variations ${ }^{11}$. Dès lors que l'on désigne, que l'on met quelque chose à distance, dès lors que l'on installe une différence, l'on est comme pris dans un double jeu entre d'une part, l'écartement de la chose et donc l'autonomisation dudit système, valant ainsi pour lui-même, et, d'autre part, cet éloignement même qui marque inévitablement l'acte de formation autant du système que de la chose.

Cette dynamique, ce mouvement des figures entre signes et monde, remarque Lyotard, est partagée par la langue aussi bien que par la perception si, comme le dit Renaud Barbaras, "approcher, c'est é-loigner, c'est-à-dire sortir de l'éloignement : la proximité conserve la distance qu'elle surmonte [...] le sentir [...] est déploiement d'une distance, [...] il n'y a donc plus d'alternative entre l'intériorité de l'expérience et l'extériorité du monde perçu" ${ }^{12}$. C'est tout d'abord cette dunamis qui caractérise le figural ou, pour mieux dire, l'espace figural, dont la figure n'est qu'une de ses déclinaisons et non pas de ses manifestations. Le figural, en effet, en agissant en tant qu'opérateur de la variation et de la différenciation, n'est pas une chose, pas non plus une essence, mais l'activité événementielle, émergente qui forme les choses reconnaissables ainsi que les actes de parole tout en y dévoilant, d'un côté, leur caractère singulier et transitoire et, de l'autre, leur façon d'être trace revenante des tréfonds des sujets qui les expriment ainsi que des cultures qui les archivent.

En d'autres termes,

le langage exerce un certain effet d'appareillage en insérant les choses dans son réseau de significations. [...] Par contre le figural c'est l'aspect sensible qui se trouve exclu des choses par ce discours, ce système langagier. Une fois que la chose sera considérée par un système selon le propre point de vue de celui-ci, elle sera traitée comme un élément de ce système et les propriétés incompatibles avec ce dernier passeront pour inaperçues. On peut dire, en caricaturant les termes, que ces propriétés inaperçues des choses par un discours constituent ce qui reste sensible dans celles-ci et font l'objet de l'espace figural. Mais, cette distinction n'est pas assignable dans les limites d'une simple opposition entre le discours et le sensible. ${ }^{13}$

L'imbrication intime du dicible et du visible exprimée par le figural et la puissance d'altérité et d'hétérogénéisation que chaque figure exemplifie comme dans le cas du jeu métaphorique, s'affichent clairement lorsque l'auteur affirme que

11 Voir notamment l'examen de l'oeuvre mallarmienne dans le texte cité.

12 BARBARAS 2009, 97.

13 SARIKARTAL 2010, 8-9.

Metodo. International Studies in Phenomenology and Philosophy

Vol. 3, n. 1 (2015) 
la figure est dehors et dedans [...] Le langage n'est pas un milieu homogène, il est scindant parce qu'il extériorise le sensible en vis-à-vis, objet, et scindé parce qu'il intériorise le figural dans l'articulé. L'oeil est dans la parole puisqu'il n'y a pas de langage articulé sans l'extériorisation d'un 'visible', mais il y est encore parce qu'il y a une extériorité au moins gestuelle, 'visible', au sein du discours, qui est son expression. $^{14}$

Et c'et dans ce miroitement ou, pour mieux dire, dans cette dynamique de transposition, $\mathrm{qu}^{\prime \prime}$ "en face du discours, il y a la figure-image" et "dans le discours, il y a la figure-forme"15, le figural articulant ainsi ses "niveaux de figurabilité", suivant la lecture d'Herman Parret, dans une tripartition de ses figures : figure-image, figure-forme et figure-matrice, cette dernière étant vue comme "ce qui ne peut être ni lu ni vu, relevant d'une topologie fantasmatique qui traverse les espaces perceptifs et oniriques sans jamais s'y montrer. Elle est la différence originaire brouillant les oppositions qui structurent tout espace, la figure topologique qui agite le désir, la disposition énergétique qui précède à tout travail de production"16.

Bien que cette tripartition soit peut-être trop tributaire de l'influence d'une vision psychanalytique du langage - la singularisation de l'événement figural procédant "uniquement" de la force pulsionnelle du désir -, il n'en reste pas moins qu'elle nous livre des suggestions fructueuses pour penser le figural en sémiotique et repenser les raisons de sa résistance.

Dans le sillage de Lyotard et suivant également l'héritage de la phénoménologie, Laurent Jenny dans La parole singulière systématise davantage les aspects saillants du figural et ses modes opératoires dans le discours :

appelons donc 'figural' le processus esthético-sémantique qui conditionne la reconduction du discours à la puissance de l'actualité. [...] Quant à la définition du figural comme 'processus esthéticosémantique', elle implique que s'y trouvent noués des processus tensionnels et des processus représentatifs ${ }^{17}$.

Définir ainsi le figural revient à affirmer non seulement la nature mixte de la langue, sa continuité avec le sensible, mais également son activité de (ré)création de formes en tant que son mode de fonctionnement 'ordinaire'. En

14 LYOTARD 1971, 13-4.

15 LYOTARD 1971, 51.

16 PARRet 2006, 108.

17 JENNY 2009, 14-15.

Metodo. International Studies in Phenomenology and Philosophy

Vol. 3, n. 1 (2015) 
effet, comme le dit Jenny, il y a

une événementialité 'ordinaire' du discours où le figural est déjà impliqué si l'on veut bien admettre qu'il recouvre tout le champ des interactions entre forme linguistique et forme non-linguistique. On voit donc qu'il intervient déjà sur un plan décisif qui n'est autre que celui de la 'réalisation' de la langue"18, il "contraint à reconsidérer la forme même de la langue [...] il nous ramène à une décision 'première' sur la forme de cette langue, il nous en représente le moment d'émergence et d'arbitraire. ${ }^{19}$.

Dès lors, montrer le caractère institué de la langue en même temps que la singularité de la situation d'énonciation qui transpose continûment des motifs perçus en des répertoires thématiques plus ou moins stabilisés au travers de l'activité mémorielle des discours attestés et gérés par la vocation identitaire et éthique des cultures, signifie interpréter la constitution des sujets énonçants et des 'objets-énoncés' comme les résultantes d'une polémique des forces, des valences perceptives concourant à la formation de valeurs. De surcroît, cela veut également dire que, derrière même les oppositions que l'on peut repérer dans le faire du sens, il y a toujours un jeu différentiel du semblable et du dissemblable faisant en sorte que les valeurs ne puissent se constituer que dans des zones de pertinence locale en même temps que générique toujours inscrites dans des champs plus vastes où elles passent au crible, entre autres, des habitus, des traditions, des domaines, etc. De ce fait, affirme Jenny, "le champ figural est plutôt un champ d'individuation que d'unification. Une tension qui 'répond' au monde et renvoie à lui se module différemment [...], un dispositif dont aucun élément ne doit être abstrait et traité pour lui-même. Il ouvre un champ différentiel plus vaste que celui où la langue s'inscrit et comme antérieur à lui." ${ }^{20}$

La notion de champ de forces - où ces forces sont à la fois de nature perceptive, linguistique, culturelle et imaginaire - se constituant conjointement à l'émergence du sens et des sujets, et celle du figural comme énergie propre de ce champ, trouvent un écho dans la théorie des formes sémantiques de Pierre Cadiot et Yves-Marie Visetti et notamment dans le concept de motif comme l'un des régimes ou phases par lesquels le sens se produit. Les auteurs s'inscrivent dans la reprise de la tradition phénoménologique aussi bien que de la Gestalttheorie précédemment mentionnée. Les acquis de la psychologie de la

18 JENNY 2009, 19.

19 JENNY 2009, 22.

20 JENNY 2009, 65 et 76 .

Metodo. International Studies in Phenomenology and Philosophy

Vol. 3, n. 1 (2015) 
forme se voient notamment complexifiés par rapport à certaines idées reçues qui installent une équivalence sinon une identité entre forme et structure impliquant par conséquent une vision close, délimitée et uniquement topologique de la forme - ou qui ne conçoivent la stabilisation d'un scénario figuratif, sa configuration, qu'en les termes d'une recomposition effectuée à partir de l'extraction de certains traits. Par contre, l'introduction du motif comme germination des valeurs - une germination toujours à régime suivant Pierluigi Basso Fossali, à savoir toujours mêlée à la présence des scènes proprement fictives, des narrations déjà en cours -, permet de mieux rendre compte de la généricité figurale en tant que dynamique énergétique façonnant la tension vers la forme. Les motifs, argumentent les auteurs,

sont en effet des unités de couplage, hautement instables, entre des dimensions qui n'apparaissent comme hétérogènes qu'à d'autres niveaux de stabilisation. Ces unités sont construites, non pas seulement par abstraction, mais aussi par des processus analogues à des synesthèses : des systèmes de transactions, de renvois multiples entre valeurs qui s'entreexpriment, ce qui est une clé essentielle pour comprendre la variété des profils obtenus. C'est en oubliant ce caractère transactionnel que l'on tombe dans les réductions configurationnelles. Il nous faut pointer, au contraire, vers une théorie de la perception qui soit une théorie motrice et intentionnelle, une théorie sémiotique et transactionnelle de l'espace, constituée par la saisie concomitante de valeurs praxéologiques, subjectives, axiologiques ${ }^{21}$.

Les motifs étant ainsi conçus, en jaillit une vision totalement autre autant des relations entre expression et contenu que de la figurativité. Du moment où la perception est d'emblée "une perception sémiotique, une perception qui se constitue comme relation à, accès vers, chemin pour, une perception d'identités qualitatives et de valeurs, qui discerne corrélativement [...] des motifs d'agir et des mouvements expressifs : ceux du sujet, ceux d'autrui, ceux de la chose même" en étant fondée dans l'activité, dans "une mise en perspective, et une suggestion d'enchaîner" ${ }^{\prime 22}$; du moment où "les formes dont il s'agit sont socialement constituées [...] à chercher dans une intersubjectivité" ${ }^{23}$, il en résulte que : i) la praxis énonciative ne se voit plus uniquement comme la mise en présence, la mise en devenir de la part d'instances, soient-elles plurielles, des virtualités du système du discours et de la signification stockées dans la

21 CADIOT, VISETTI 2002. Pour un examen global de la théorie des formes sémantiques, voir: CADiot, VisetTi 2001.

22 CADIOT, VisetTI 2002, 16.

23 CADiOT, VisETTI 2002, 19.

Metodo. International Studies in Phenomenology and Philosophy

Vol. 3, n. 1 (2015) 
mémoire culturelle, mais elle devient le lieu réflexif d'appréciation, d'ajustement, d'évaluation, de négociation, de (re)institution - dans la monstration précisément de leur caractère institué - de ces mêmes significations et ii) la figurativité récupère le caractère intermédiaire de la figura, en se situant transversalement par rapport aux différentes substances signifiantes et permettant ainsi une transduction, une remédiation des formes de leur même apparaitre. Dès lors, le figural agit, en traversant le dicible, le visible et le sensible, comme un imaginaire "des corps-choses et de leurs empreintes, un imaginaire des affects et des champs d'action [...] toujours présent-absent", comme "l'ouverture surabondante du sensible, en même temps que son retrait, devant nos tentatives d'appréhension, de délimitation, de catégorisation" ${ }^{24}$. Passons ainsi à examiner les imbrications entre le figural/imaginaire formulé de cette façon et ses implications pour une sémiotique qui se veut véritablement perceptive.

\section{Figural, "magma" et sémiotique de la perception}

Si l'on veut comprendre pleinement le rôle de la dimension figurale à l'oeuvre dans les processus de sens, un périple autour de la pensée du philosophe et psychanalyste franco-grec Cornelius Castoriadis et plus particulièrement autour de ses concepts de magma et d'imaginaire radical/imaginaire social s'impose. En étudiant le changements des sociétés et en se questionnant sur l'existence de soi-disant principes ou de tendances qui pourraient rendre compte historiquement de ces changements - son élaboration étant issue à partir d'une critique du marxisme -, Castoriadis, dans une tentative de dépassement des explications fonctionnalistes en sociologie, va à la rencontre d'une absence de fondement spécifique qui puisse avoir le dernier mot quant au 'pourquoi' d'une telle évolution plutôt que d'une autre. Les activités humaines paraissent en effet ne pas trouver des véritables justifications ou des fins dans des marco-configurations sémiotiques telles l'économie, la politique, etc. ; au contraire, souligne Castoriadis, ce que les différentes formes symboliques constituant les sociétés - et il en va de même pour les significations - partagent entre elles, c'est le fait d'être issues d'un processus de création qui les institue en tant que telles et qui, une fois les ayant instituées, leur confère une existence, les fait être.

Les actes de création/institution sont à la fois ce qui permet à une société

24 VISETTI, 2014.

Metodo. International Studies in Phenomenology and Philosophy

Vol. 3, n. 1 (2015) 
donnée et à des individus de se penser en tant que tels et la dynamique propre et interne aux sociétés et aux individus. Plus précisément, la création se produit par l'activité imaginante des individus et des sociétés manipulant, modelant les significations données en partant de la perception jusqu'à préfigurer l'insurgence du nouveau par la représentation. C'est la raison pour la quelle la création est ex nihilo sans que cela veuille dire qu'elle le soit

in nihilo (dans le rien) [...] ni cum nihilo (sans moyens ni conditions). Autrement dit, comme l'écrit Castoriadis, 'il est clair que la création social-historique (comme du reste dans n'importe quel autre domaine), si elle est immotivée - ex nihilo - a toujours lieu sous contraintes. $\mathrm{Ni}$ dans le domaine social-historique ni nulle part ailleurs, la création ne signifie pas que n'importe quoi peut arriver n'importe où, n'importe quand et $n^{\prime}$ importe comment ${ }^{25}$.

L'imaginaire, qu'il soit 'radical' lorsqu'il concerne un single individu ou social lorsque l'on a à faire avec des sociétés entières, régit la création en se constituant ainsi, comme le souligne Antonino Bondì, comme "la clef de voûte pour penser la nature humaine et ses modalités de se mettre en contact avec le monde" ${ }^{26}$, comme

une faculté typiquement humaine de déplacement, transposition et création, dont le but est de construire une sorte d'atmosphère ou de milieu mobile dans lequel tout ce qui est perçu n'est pas seulement la trace d'une activité expressive, mais aussi le résultat $\mathrm{d}^{\prime}$ une fabrication sociale, d'un enrôlement conflictuel, d'un dispositif de mise en place d'une scène interlocutoire extrêmement complexe et gérée par des acteurs sociaux doués d'un statut social spécifique ${ }^{27}$.

Or, la condition par le biais de laquelle l'imaginaire peut exercer son faire réside précisément en le fait que les formes sémiotiques, les significations, sont par leur même facture transposables, perméables, qu'elles constituent, suivant Castoriadis, un magma. Le caractère toujours à déterminer du magma, appelé également boue sémantique, préside notamment autant à la détermination d'ensembles de significations et d'identités déterminées historiquement qu'à la possibilité de faire émerger, du fond du temps, l'inattendu - et c'est toute là sa puissance figurale -, le nouveau, le à venir des significations. Comme l'auteur l'explique dans son oeuvre majeure, L'institution imaginaire de la société, "l'institution de la société est chaque fois institution d'un magma de 25 TOMÈs 2007, 79-80.

26 BONDì 2014, 5 .

27 BONDİ 2014, 5 .

Metodo. International Studies in Phenomenology and Philosophy

Vol. 3, n. 1 (2015) 
significations imaginaires sociales, que nous pouvons et devons appeler un monde de significations ${ }^{\prime 28}$ et

en tant que magma, les significations [...] ne sont pas des éléments d'un ensemble soumis à la déterminité comme mode et critère d'être. Une signification est indéfiniment déterminable [...] sans que cela veuille dire qu'elle est déterminée. Elle peut toujours être repérée, assignée provisoirement comme élément identitaire [...] Mais ces déterminations ne l'épuisent, par principe, jamais ${ }^{29}$.

Ce qui caractérise l'activité imaginaire et les fluctuations du magma - et c'est ce qui représente l'originalité de la pensée de Castoriadis - c'est le fait que la dynamique instituante non seulement reconfigure la trame des valeurs des individus et des sociétés, pour ainsi dire, dans l'abstrait, mais elle opère précisément dans et à partir de la perception - culturelle - en travaillant les formes-figures des objets sémiotiques car, comme poursuit le texte, "l'institution du monde des significations comme monde social-historique est ipso facto 'inscription' et 'incarnation' dans le 'monde sensible'" ${ }^{30}$. Les actes de création/institution mettent à l'épreuve la teneur des valeurs dans le temps, ce qui revient à affirmer "l'auto-altération perpétuelle de la société [...] qui se manifeste par la position de formes-figures relativement fixes et stables et par l'éclatement de ces formes-figures qui ne peut jamais être que position-création d'autres formes-figures" ${ }^{31}$.

Dans la perspective d'une sémiotique de la perception et des cultures à vocation écologique, telle qu'elle est promue par Basso Fossali, cela revient à considérer la dimension figurale du sens comme "le carrefour des échanges de sens entre mondes. En effet le rôle anthropologique détenu par le figural dépend du fait que le monde de l'expérience, ainsi que chaque monde fictif élaboré à des buts explicatifs, ne peuvent jamais garantir une totalisation du sens $^{\prime \prime 2}$. Dans la conception et dans le modèle de cet auteur, il y a, à partir de la perception déjà informée par la culture, une démultiplication des accès au sens qui s'effectue par un va-et-vient de médiations entre l'expérience et l'existence, entre la sensibilité et l'affectivité et les procès de textualisation, entre les cours d'action et leur instabilité et les narrations, les configurations identitaires qui les gèrent dans le temps. Les différentes médiations représentent, pour

28 CASTORIADIS $1975,480$.

29 CASTORIADIS 1975, 465.

30 CASTORIADIS $1975,475$.

31 CAstoriadis 1975, 496.

32 Basso Fossali 2006, 19.

Metodo. International Studies in Phenomenology and Philosophy

Vol. 3, n. 1 (2015) 
simplifier, autant de "lectures", de pratiques de surreflexion de la part des sujets, de réflexion de la part des textualisations, au cours même des expériences ou de l'appréhension des textes. Les plis des différentes lectures lors de l'énonciation remettent en cause, en les complexifiant, la tension vers la figure propre de toute perception. Emérgeant de l'appréhension perceptive, un plan figuratif peut se constituer, ouvrant à un nouveau passage de la sémantisation, à partir duquel Basso Fossali dénombre au moins trois lectures, trois modes de constitution du sens d'un texte, à savoir : 1) une "lecture plastique de l'énonciation plastique "lorsque l'énoncé s'autonomise en tant que "pur ensemble de pattern" ;2) une "lecture figurative d'une énonciation plastique" lorsque l'on récupère une figurativité par "la mise en valeur d'une mémoire discursive" ; 3) une "lecture figurale" lorsque le plastique entre "en tension avec la stabilisation du scénario figuratif" ${ }^{\prime 33}$.

Le figural, notamment dans les images - et c'est pourquoi l'auteur se focalise sur l'image audiovisuelle et cinématographique - se pose comme "une mise en perspective du matériau discursif qu'en met l'emphase sur les relations internes, en particulier en termes de tension entre d'éléments plastiques et d'éléments figuratifs" ${ }^{\prime 34}$. Ce faisant, l'activité figurale non seulement remotive "les choix expressifs d'un texte" en sa globalité - en faisant bouger par le même coup tout le champ dans lequel ledit texte s'inscrit -, mais il occasionne également, au moins dans les textes filmiques, une "réfraction continue du niveau de l'énoncé vers celui de l'énonciation" ${ }^{35}$. De ce fait, on retrouve ici le fil rouge traversant les différentes formulations du figural précédemment évoquées.

A l'instar des suggestions de Castoriadis, le figural non seulement traverse les espaces énonciatifs et les matières de la perception et de la fiction, en les multipliant par la création d'un espace tiers de tension entre les valences aussi bien que les valorisations, mais il s'avère, selon l'auteur, dans le basculement des formes-figures,

l'épiphénomène de l'observation de second ordre sur la signification ; on joue un match, mais en même temps on observe la manière dans laquelle les joueurs observent ces règles, on tient un discours mais en même temps on le fait se replier pour qu'il réfléchisse sur sa propre licéité quant à la manière dans laquelle il est énoncé. [...] Le figural est toujours une sorte de 'spasme' interprétatif instituant des relations entre les fronts les plus opposés de la signification textuelle, de la

33 BASSO FossaLi 2009, 27.

34 Basso Fossali 2003, 29.

35 Basso Fossali 2003, 30.

Metodo. International Studies in Phenomenology and Philosophy

Vol. 3, n. 1 (2015) 
réémergence de la sensibilité de la signification à la pertinetialisation culturelle la plus sofistiquée. ${ }^{36}$

Dès lors, le figural en tant qu'imaginaire en action, oblige à garder l'indécision et le mouvement entre des lectures, en tant qu'indécision productrice de sens en elle-même.

A ce propos, et à titre purement d'exemplification, nous nous attardons très brièvement sur deux oeuvres de l'artiste italien Alighiero Boetti, ayant fait partie du courant artistique connu aux années soixante sous le nom d'Arte povera (Art pauvre) et ayant par la suite suivi un cheminement tout à fait personnel.

En particulier, les oeuvres qui retiennent notre attention sont : Shaman Showman, lithographie de 1968 (fig. 1) et la série de tapisseries Tutto (Tout) fabriquées entre 1980 et 1988 (fig. 2).

36 Basso Fossali 2006, 18.

Metodo. International Studies in Phenomenology and Philosophy

Vol. 3, n. 1 (2015) 


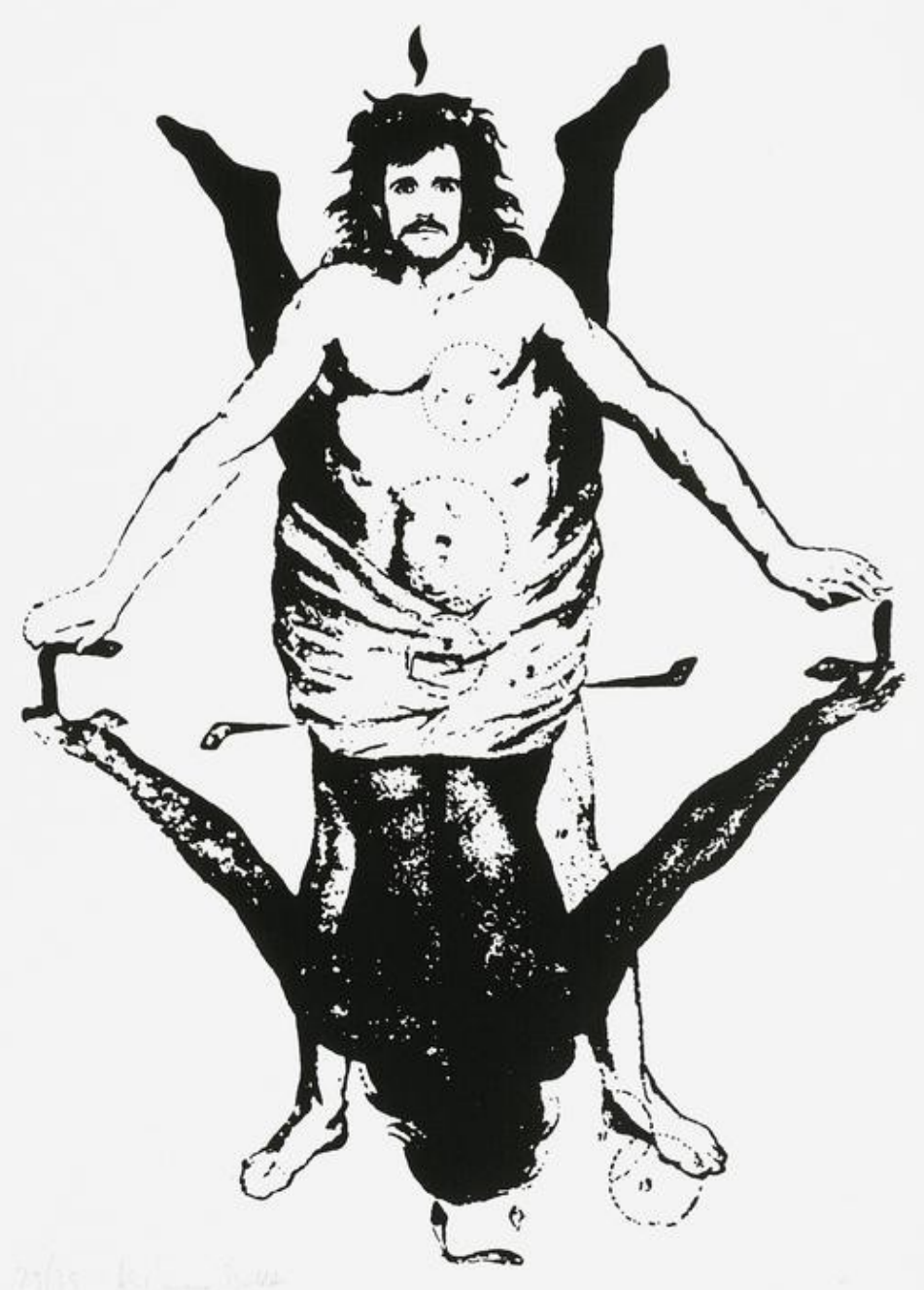

Fig. 1 Alighiero Boetti, Shaman Showman, 1967, Galerie de Nieubourg, Milan

Metodo. International Studies in Phenomenology and Philosophy Vol. 3, n. 1 (2015) 


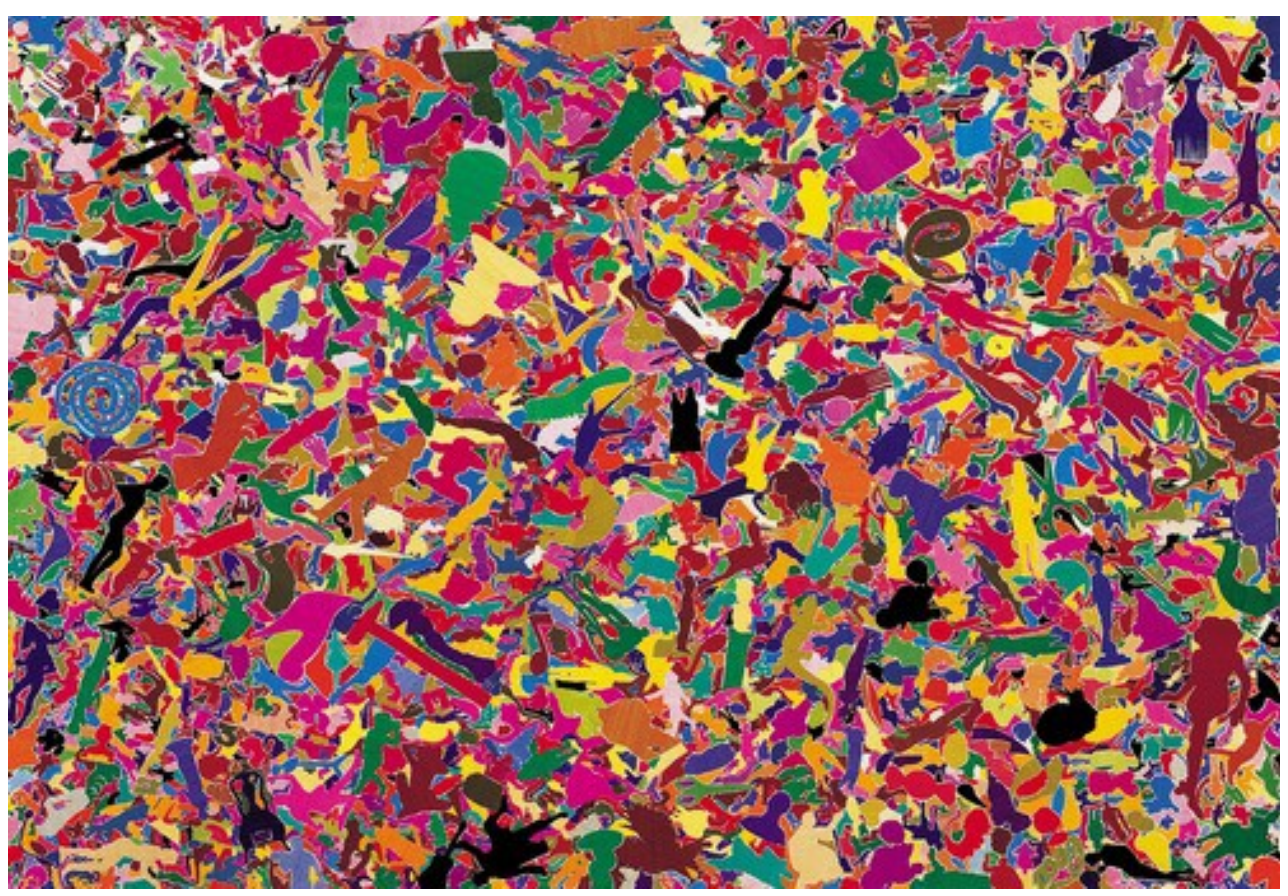

Fig. 2 Alighiero Boetti, Tutto, 1987, Centre Pompidou, Paris

Travaillant la démultiplication aussi bien linguistique - il avait scindé pour les re-associer son nom et son prénom en se baptisant artistiquement Alighiero e Boetti - que matérielle et sensible, - en se servant de matériaux trouvés ou d'objets du quotidien -, Boetti défie, dans les deux oeuvres en question, la dualité des catégorisations, des oppositions et des lectures par le biais de procédés similaires de monstration de la production de la différence. Dans Shaman Showman par exemple, il opère tout d'abord une répétition, un doublement de la figure, - son autoportrait - en se servant d'un principe de symétrie ; l'image se trouve ainsi rebattue sur elle-même comme le négatif de la première. Néanmoins, le rebattement s'écarte de la géométrisation de l'espace dès lors que l'image rebattue se montre comme figure renversée. C'est là que le visage, ayant fondé au début la possibilité de reconnaitre l'image, la figure entière en tant qu'autoportrait, se tourne vers le fond de l'affiche, à savoir vers le dessin s'entrevoyant à peine, et cède ainsi la place aux densités chromatiques - les concrétions noires faisant estomper les contours, les limites anatomiques de la forme humaine. Comme le remarque Georges Didi-Huberman dans son Phalènes, où le papillon exemplifie et se fait imago du trouble figural qui gîte dans les images, "dire de l'imago qu'elle 'bat des ailes', c'est dire que sa

Metodo. International Studies in Phenomenology and Philosophy 
symétrie même est une danse : tous ensemble liberté du corps et péril dislocation, fente, destruction - pour sa propre forme. [...] Toute symétrie est en attente de l'événement qui la disloquera d'un coup [...] Il suffit par exemple de symétriser un visage pour le rendre inhumain, voire méconnaissable ${ }^{\prime 37}$. De ce fait, c'est la constitution même de la forme, dans une reprise d'un motif que l'on pourrait qualifier de rébus à l'instar des formes des tests perceptifs de Rorschach, qui engage un agonisme et des aller-retour entre une lecture figurative et une lecture plastique. En effet, l'exploration énigmatique proposée est renforcée par une mise en mouvement du même processus de lecture ; aux quatre angles formés par le doublement de la figure initiale ainsi que dans le corps de l'artiste - mais uniquement le corps rendu visible en son intérieur par le blanc de l'image -, l'on voit et l'on reconnait en filigrane des signes graphiques de nature double, des lettres hébraïques aux vertex et, à l'intérieur du corps, des diagrammes, des circonférences. Il s'agit de l'Adam Kadmon, l'"homme originel", terme cabalistique issu du symbolisme du Zohar, qui exemplifie, à travers les correspondances installées entre les parties du corps humain et les cercles des différents sefirot de l'Arbre de Vie - les hypostases émanées du divin -, l'interprétation cabalistique de l'imago dei, c'est-à-dire de la création de l'homme à la ressemblance de Dieu. Ne pouvant pas creuser davantage l'analyse de la litographie, soulignons tout de même que, déjà d'une prime abord, il est ici question d'une dynamique de passages du rébus à l'altérité (le doublement imparfait) à la ressemblance (à Dieu) à la différence des signes et leurs "énergie sémantique" ${ }^{\prime 38}$, plutôt que d'oppositions entre, par exemple, haut/bas, blanc/noir ${ }^{39}$ etc...

Avec la tapisserie Tutto l'action du figural en tant qu'opérateur d'indiscernabilité et de transposition entre substances, entre genres, etc, se montre davantage jusqu'à s'étaler sur tout le volume - plutôt que la surface constituant l'espace de l'image. Tutto est une série de tapisseries (arazzi) datant des années 80 qu'Alighiero Boetti fit fabriquer au Pakistan par des femmes artisanes selon une méthode traditionnelle qu'il découvrit lors d'un voyage en Afghanistan en 1975. Tutto affiche sans l'épuiser toute la richesse d'une lecture figurale et matérique à la fois, dans laquelle figurativité et plasticité se

37 DiDi-HubERMAN 2013, 58 et 60.

38 Voir à ce propos OUAKNIN 2003.

39 Et il ne pourrait qu'être ainsi, du moment où dans la Table d'émeraude, l'un des plus célèbres textes de la littérature alchimique, on lit que "Ce qui est en bas, est comme ce qui est en haut; et ce qui est en haut est comme ce qui est en bas, pour faire les miracles d'une seule chose". L'Hortulain (Ortulanus), vers 1350 : La table d'émeraude d'Hermès Trismégiste, avec les commentaires de l'Hortulain, Éditions Traditionnelles, Paris, 2000.

Metodo. International Studies in Phenomenology and Philosophy

Vol. 3, n. 1 (2015) 
disputent l'espace selon des degrés de distanciation physique stricto sensu de l'acte de lecture, de parcours, d'exploration de l'oeuvre. Au doublement de Shaman Showman se substitue ici un procédé mimétique d'un magma, d'un mélange sans début ni fin où est abolie toute forme de symétrie à l'intérieur de l'oeuvre, remplacée par la symétrie frontale de l'espace extérieur de sa perception. Par conséquent, dans le va-et-vient des mouvements d'éloignement et de rapprochement, se crée une épaisseur de la vision - une vision dans ces cas-ci véritablement haptique - qui, en élargissant son extension, fait déborder les contours du statut de l'oeuvre, du visuel jusqu'aux bordures d'une sculpture textile. Et c'est précisément le mouvement qui permet d'alterner, en les gardant constamment en tension, une tension qui se fait musculaire, les deux lectures car ce qu'il apparait à une première vue semble être une masse de formes et de chromatismes divers pour se configurer, en regardant de près, comme un enchevêtrement de figures : des silhouettes humaines et animales, des esquisses de visages, des profils d'objets. De surcroît, un autre aspect relevant d'une dynamique figurale en action, légitime le maintien des deux lectures, à savoir l'effet de saturation engendrant l'inextricabilité de la vision. Il est obtenu, comme en transposant toujours une sorte de rébus perceptif de l'image à sa technique de production, dans ce cas le travail du tissu, par l'institution d'un principe arbitraire, c'est-à-dire le choix de confier aux artisanes la même longueur de fil pour chaque couleur.

Tutto parait ainsi mimer et incarner, dans les chaînes et dans les trames, dans les plis de la tapisserie, le mode opératoire même par lequel fonds et formes coémergent.

\section{Conclusion}

Pour résumer, ayant passé en examen de nombreuses facettes du dispositif figural, à la fois ponctuel dans ses effets aussi et difficilement saisissable au préalable $\iota_{\iota}$ procédons maintenant à une récapitulation de celles qui nous paraissent être ses caractéristiques les plus saillantes afin d'esquisser tant une problématisation de la théorie qu'une opérationnalité à l'égard de l'analyse sémiotique. Dès lors, le figural, nous semble-t-il, concerne et investit :

- une dimension énergétique (ex : investigation, également dans le sillage de la sémiotique tensive, des relations des forces et de leur constitution diagrammatique) ;

- une dimension d'altérité (ex : investigation des tensions éventuelles entre

Metodo. International Studies in Phenomenology and Philosophy

Vol. 3, n. 1 (2015) 
rôles à différents niveaux, de l'expérience aux pratiques sociales) ;

- une dimension émotionnelle (ex : émergence des émotions et reconfiguration d'un cours d'action) ;

- une dimension de médialité (ex : saisie des traces d'une activité figurale lors des passages entre une médiation et l'autre ou lors des passage entre des objets sémiotiques médiatisés par leur propre constitution, question de la transposition de formes et motifs) ;

- une dimension temporelle (ex : émergence et survivance, question du changement culturel, révision du modèle lotmanien et dialectique des images des formes de vie à la lumière de l'imaginaire) ;

- une dimension d'étendue (ex : relation entre activités singularisantes et dynamiques globales, questionnement et remaniement des notions telles le genre, le style, etc.)

L'enquête que nous avons jusqu'à ici menée sur les modes de fonctionnement $\mathrm{du}$ figural ainsi que les volets que l'on a dégagés en relation avec des problématiques exquisément sémiotiques, frayent une voie à l'élargissement de la portée analytique du figural à d'autres phénomènes de sens. En effet, tout au long de cette traversée, l'on a pu remarquer que la dimension figurale est émergée à partir d'études portant tantôt sur le discours linguistique, tantôt sur les images, tantôt sur des analyses comparant ces deux objets. Le prolongement de ces questionnements concernera l'approfondissement des relations entre dimension figurale et proccesus de subjectivation, à savoir entre figuralité - en tant qu'épaisseur perceptive et culturelle de l'apparaître - et, en dernière analyse, l'intentionnalité conçue comme "un sens d'être spécifique, qui est celui d'un dynamisme" ${ }^{\prime 40}$, autrement dit comme mouvement.

\section{Bibliographie}

Aubral, F. \& ChÂTEAU, D. 1999. Figure, figural. Paris: l'Harmattan.

AuERBACH, E. 1968. Mimésis. Paris : Gallimard.

- 1993. Figura. Paris :Belin.

BARBARAs, R. 2009. La perception. Essai sur le sensible. Paris : Vrin.

40 Barbaras 2003, 14.

Metodo. International Studies in Phenomenology and Philosophy

Vol. 3, n. 1 (2015) 
- 2003. Vie et intentionnalité. Paris : Vrin.

BAsso Fossali, P. 2002. Il dominio dell'arte. Semiotica e teorie estetiche. Roma: Meltemi.

- 2003. Confini del cinema. Strategie estetiche e ricerca semiotica. Torino : Lindau.

- 2006. Interpretazione tra mondi. il pensiero figurale di David Lynch. Pisa : ETS.

- 2009. La tenuta del senso. Roma: Aracne.

Bondì, A. 2012. Percezione, semiosi e socialità del senso. Milano : Mimesis.

- 2014.«De la valeur au magma ou de Saussure à Castoriadis. Théorie(s) sémiologiques et théorie de l'esprit », Online document: Formes symboliques, http://formes-symboliques.org/spip.php?article322

CAdiot, P. \& Visetti, Y.-M. 2001. Pour une théorie des formes sémantiques. Paris : PUF.

- 2002. "Motifs linguistiques et construction des formes sémantiques schématicité, généricité, figuralité», in LAGOrGETte, D., LARRIVÉE, P. (eds) (2002), Representations $d u$ Sens linguistique, LINCOM Europa, LINCOM Studies in Theoretical Linguistics.

CAstoriadis, C. 1975. L'institution imaginaire de la société. Paris: Éditions du Seuil,.

- 1986. Domaines de l'homme. Les carrefours du labyrinthe 2, Éditions du Seuil, Paris.

- 1997. Fait et à faire. Les Carrefours du labyrinthe 5. Paris : Éditions du Seuil.

- 2007. L'imaginaire comme tel. Texte établi, annoté et présenté par Arnaud Tomès. Paris : Hermann.

CORRAIN, L., VALENTI, M. (ed) 1991. Leggere l'opera d'arte. Dal figurativo all'astratto. Bologna : Esculapio.

Didi-Huberman, G. 2002. L'image survivante. Histoire de l'art et temps des fantômes selon Aby Warburg. Paris : Les Éditions de Minuit.

- 2013. Phalènes. Essais sur l'apparition, 2. Paris : Les Éditions de Minuit.

- 2014. Sentir le grisou. Paris : Les Éditions de Minuit.

ECO, U. 1975. Trattato di semiotica generale. Milano : Bompiani.

- 1985. Sugli specchi e altri saggi. Il segno, la rappresentazione, l'illusione, l'immagine. Milano : Bompiani.

Fontanille, J. 2003. Sémiotique du discours, Limoges : Pulim.

- 2004. Figure del corpo. Per una semiotica dell'impronta. Roma :Meltemi.

- 2008. Pratiques sémiotiques. Paris: PUF.

Fontanille, J. \& Tore, G. M. 2006. «De la modalisation à l'esthésie: considérations (in)actuelles sur le passage de Du sens à Du sens II ». Protée, 34, 23-32.

Greimas, A. J. 1984. «Sémiotique figurative et sémiotique plastique ». Actes

Metodo. International Studies in Phenomenology and Philosophy

Vol. 3, n. 1 (2015) 
Sémiotiques, 60.

Greimas, A. J., Courtés, J. 1979. Sémiotique. Dictionnaire raisonné de la théorie du langage. Paris : Hachette, .

Hermès Trismégiste, 2000. La Table d'émeraude d'Hermès Trismégiste avec les commentaires de L'Hortulain. Paris : Éditions traditionnelles.

JenNY, L. 2009. La parole singulière. Paris : Belin.

LYOTARD, F. 1971. Discours, figure. Paris : Klincksieck.

Merleau-Ponty, M. 1945. Phénoménologie de la perception. Paris : Gallimard.

- 1960. Signes. Paris : Gallimard.

- 1964. Le visible et l'invisible. Paris : Gallimard.

- 1969. La prose du monde. Paris : Gallimard.

- 2011. Le monde sensible et le monde de l'expression. Genève : MetisPresses.

OuAKNIN, M.-A. 2003. Concerto pour quatre consonnes sans voyelles. Paris : Payot.

PARRET, H. 2006. Sutures sémiotiques. Limoges : Lambert-Lucas.

SARIKARTAL, E. 2010. «Discours du figural». Appareil 6, en ligne, http://appareil.revues.org/355

TOMÈs, A. 2007. «Introduction à la pensée de Castoriadis», in CASTORIADIS, C. (2007), L'imaginaire comme tel. Texte établi, annoté et présenté par Arnaud Tomès. Paris : Hermann.

VisetTI, Y.-M. 2014. «Motifs et expressivité ». Formes symboliques, http://formes-symboliques.org/spip.php?article85

Metodo. International Studies in Phenomenology and Philosophy

Vol. 3, n. 1 (2015) 